\title{
Représentations autour du niveau de langue : le cas du français à l'université
}

Nabil Sadi, Université de Béjaia, Algérie

Le français est regardé par ses locuteurs comme une langue homogène. Pourtant, il serait bien difficile de trouver deux personnes pour le parler de façon absolument semblable ; et personne pour le parler de la même manière en toutes circonstances. (Gadet)

Cet article a pour objectif d'étudier le français parlé par les étudiants algériens. Il vise, à travers deux volets, à étudier le niveau de ces étudiants en langue française, tel qu'il est décrit par les enseignants, et sur ce qui le caractérise tant à l'oral qu'à l'écrit, ainsi que les procédés de dénomination utilisés par les enseignants pour qualifier le français des étudiants de l'université de Bejaia. Il s'agit de décrire les différents termes employés pour nommer ce français parlé, déterminer les différentes formes sous lesquelles apparaissent ces divers qualifiants et faire ressortir les facteurs qui régissent le choix de ces termes. Nous nous sommes inspiré du travail de Yamina-Chafia Benmayouf qui a identifié les différentes désignations de l'arabe à travers une enquête menée auprès d'un groupe d'étudiants du département de français de l'Université de Constantine en juin 2003.

Avant de répondre à ces questions, nous mettrons l'accent essentiellement sur le français dans l'Algérie d'aujourd'hui en retraçant son cheminement depuis l'occupation française jusqu'après l'indépendance. Nous ne manquerons pas de décrire l'aventure de cette langue dans un environnement plurilingue et d'évoquer son statut ainsi que ses domaines d'usage, sans trop insister sur ce qui caractérise la situation sociolinguistique de l'Algérie.

\section{La place du français dans l'Algérie d'aujourd'hui}

Quelques années seulement après l'occupation française, le français a réussi à s'implanter en Algérie et à s'imposer comme langue officielle. A cet égard, Gilbert Grandguillaume souligne que «si la langue française fut la langue des colons, des Algériens acculturés, de la minorité scolarisée, elle s'impose surtout comme langue officielle, langue de l'administration et de la gestion du pays, dans la perspective d'une Algérie française » (19). Après l'indépendance, la langue française reste extrêmement répandue en Algérie: «l'étendue et la diversité des champs d'action de cette langue ainsi que son prestige semblent 
être les facteurs dynamisants qui lui confèrent une bonne position dans la hiérarchie des valeurs sur le marché linguistique algérien »(Queffélec et al., 37). En effet, le français occupe une place prépondérante dans plusieurs domaines, à savoir économique, social et éducatif. Il tient aussi une position forte dans l'enseignement universitaire ainsi que dans le secteur médiatique.

\section{Le français au contact des langues locales}

Le paysage sociolinguistique en Algérie se caractérise par une situation où coexistent plusieurs langues (et variétés de langue): «En Algérie, trois langues sont parlées au quotidien ayant chacune ses quelques millions de locuteurs. Il s'agit dans l'ordre décroissant $\mathrm{du}$ nombre de locuteurs qui les pratiquent: de l'arabe ${ }^{1}$, du berbère et du français » (Cheriguen, «Quels mots », 218). L'usage de l'arabe classique est réservé essentiellement au système éducatif, à la télévision, aux actes administratifs et à une partie de la presse écrite et orale. L'arabe dialectal est la langue maternelle de la plus grande partie des Algériens. Le berbère, reconnu en 2002 comme langue nationale, se décline en plusieurs variétés dont le kabyle, langue du chaoui en usage dans les Aurès, le mozabite dans le M'zab et le targuie dans le massif du Hoggar. Quant au français, il y a un nombre important de locuteurs maîtrisant plus ou moins cette langue véhiculaire : ceux qui parlent réellement le français dans la vie de tous les jours, ceux qui le parlent occasionnellement et enfin ceux qui comprennent cette langue mais qui ne la parlent pas. S'agissant des «francophones occasionnels » qui alternent le français et l'arabe dans des situations formelles ou informelles, nous pouvons ajouter que les pratiques langagières à l'œuvre aujourd'hui en Algérie témoignent d'une instabilité plus complexe dans l'utilisation des codes linguistiques en présence, dans la mesure où un même locuteur peut passer d'un code à un autre, voire même à plusieurs dans un même énoncé, s'érigeant ainsi en véritable plurilingue.

Cette alternance codique a plusieurs raisons d'être et peut s'expliquer par plusieurs facteurs. D'une part, elle peut découler d'un écart entre le lexique français et les univers culturels ou institutionnels ${ }^{2}$. D'autre part, elle peut représenter une stratégie de communication qui renvoie à une compétence ou à une incompétence linguistique. Le locuteur, obéissant au principe du moindre effort, peut utiliser un vocable dans une autre langue, répugnant à chercher le terme équivalent dans la langue dans laquelle il s'exprime, ou bien comme l'expliquent Josiane F. Hamers et Michel Blanc «pour compenser son manque de compétence en faisant appel tantôt à une langue, tantôt à l'autre pour maximaliser l'efficacité de la communication »(203). 


\section{L'enseignement $d u$ et $e n$ français à l'université}

A partir de 1962, un enseignement $d u$ et en français s'est instauré en Algérie :

La scolarisation massive en Algérie a permis une plus grande généralisation de la langue française. Les médias, la presse orale et surtout écrite ont aussi apporté une contribution importante à la diffusion du français dans le pays. La quasi-totalité de la population née à partir de 1962 a bénéficié d'un enseignement en français, puis progressivement, d'un enseignement de français. (Cheriguen, « Politique », 66)

Mais depuis 1984, le français a perdu son statut de langue véhiculaire dans les lycées algériens. Relégué au statut de langue « étrangère », il se trouve réduit à un enseignement de la langue en tant que telle. L'enseignement de toutes les matières est dispensé en arabe, ce qui engendre un très bas niveau de français chez les futurs bacheliers, pour ne pas dire un échec pur et simple d'une partie du système éducatif algérien. En effet, chaque année, les enseignants universitaires déplorent le niveau des étudiants qui ne cesse de se dégrader.

Ces nouveaux bacheliers pourront s'inscrire dans des filières où le français est la seule langue d'enseignement. Il s'agit des disciplines scientifiques et technologiques : médecine, pharmacie, informatique, architecture, sciences vétérinaires et l'ingéniorat. Le nombre d'étudiants qui y accèdent, est minime, vu la moyenne exigée et le niveau de langue requis. Par ailleurs, leur faible niveau peut s'expliquer aussi par l'absence de l'enseignement de la langue française dans certaines régions du pays, comme le notent les auteurs du Rapport général de la commission nationale de la réforme du système éducatif: «Les insuffisances voire l'absence de l'enseignement de la langue française, par exemple et parce que censée être généralisée, a exclu une frange de la population estudiantine des études scientifiques et technologiques » (152).

D'autres suivront une licence de français qui exige un très bon niveau de langue pour assimiler les enseignements théoriques. Et enfin, il y a ceux qui choisiront un cursus arabisé dans lequel l'enseignement/apprentissage d'une seconde langue est obligatoire. Il s'agit de «filières sciences humaines ou sciences fondamentales confondues où la langue française, en tant que moyen d'accès à un savoir spécialisé, est enseignée à raison de 4 h hebdomadaire pendant les premières années universitaires » (Queffélec et al., 93).

L’Université de Béjaia est un établissement public de formation supérieure, sous tutelle du Ministère de 1'Enseignement Supérieur et de la Recherche Scientifique, structurée en sept facultés. Elle accueille, en plus de la plus grande majorité des bacheliers de la région de Béjaia, d'autres bacheliers venant d'autres régions du pays, ainsi que des étudiants étrangers, essentiellement des Africains ${ }^{3}$. 


\section{Présentation de l'enquête}

Notre enquête a été réalisée par le biais d'un questionnaire organisé autour de deux rubriques, à savoir l'identification des enquêtés (âge, sexe, profession, faculté et département d'exercice). Les quatre questions réparties en deux dimensions, à savoir le niveau de langue des étudiants à l'écrit et à l'oral (nous voulions connaître ce que pensent les enseignants du niveau de leurs étudiants en langue française), les opinions et les représentations que les enseignants ont du français parlé par leurs étudiants (nous souhaitions savoir comment est qualifié ce français parlé et ce qui le caractérise et, par conséquent, déterminer les différentes nominations que recouvre ce français parlé).

Nous avons proposé ce questionnaire à des enseignants de deux facultés : la faculté des lettres et des sciences humaines et la faculté des sciences économiques, sciences de gestion et sciences commerciales. Notre objectif était de cibler des filières caractérisées par l'enseignement $d u$ et en français, à savoir les départements de français, de sociologie, de berbère, des sciences économiques, de gestions et des sciences commerciales. Le fait d'avoir sollicité des enseignants qui travaillent dans une autre faculté ou dans un autre département, nous a posé quelques problèmes. Nous avons distribué 50 questionnaires et nous n'avons pu en récupérer que 44.

\section{Le niveau des étudiants}

Sur le niveau des étudiants à l'oral, les enseignants ont répondu à la question : «Que pensez-vous du niveau en français de vos étudiants à l'oral ? »Ils ont eu à choisir entre «bon », «assez bon », « mauvais » et «très mauvais ». Nous avons noté que 21 enseignants contre 23 estiment que les étudiants sont assez bons ${ }^{4}$ à l'oral. Ils jugent que les étudiants s'expriment bien, qu'ils ont plus de facilité à parler qu'à écrire. Selon eux, les étudiants sont influencés par les médias et les nouveaux moyens de communication, ce qui explique qu'ils sont à l'aise, s'expriment facilement et correctement et arrivent à formuler des phrases correctes et cohérentes. Les enquêtés qui considèrent que les étudiants sont mauvais à l'oral, n'omettent pas de signaler qu'il y a des exceptions. Ils trouvent que la majorité des étudiants ne prononcent pas correctement et qu'ils réfléchissent en kabyle ou en arabe dialectal pour construire des bribes de phrases en français. Ils font tous référence aux problèmes de conjugaison, de syntaxe et de vocabulaire qui renvoient à un français approximatif.

Concernant l'écrit, la même question a été posée aux enseignants et la même catégorisation leur a été proposée, à savoir choisir entre «bon », « assez bon », « mauvais » et «très mauvais ». Nous avons constaté que presque la totalité des enquêtés (39 enseignants 
contre 5) qualifient le niveau des étudiants de mauvais, voire de très mauvais ${ }^{5}$. Les 39 enseignants s'accordent à dire qu'il existe un grand décalage entre l'écrit et l'oral et que très peu d'étudiants maîtrisent le code écrit. Ils conviennent que la majorité ne maîtrise pas la grammaire ainsi que l'orthographe et les règles élémentaires de l'écriture, conséquences, selon eux, des SMS et du tchat. Les enseignants soulèvent à plusieurs reprises des difficultés qui portent sur la cohérence et la cohésion du texte ainsi que sur l'organisation du discours. Par ailleurs, cinq enseignants partagent l'idée que les étudiants n'ont pas tous le même niveau à l'écrit et qu'il y a de très bons étudiants dont les écrits se distinguent largement. Néanmoins, nous relevons un paradoxe dans leurs explications, à savoir qu'ils considèrent également que les écrits des étudiants sont souvent caractérisés par des fautes d'orthographe et une mauvaise formulation des phrases.

Un autre paradoxe est à signaler; en dépit de la bonne appréciation du niveau des étudiants à l'oral par 21 enseignants sur 44, cela n'empêche pas 20 d'entre eux de qualifier péjorativement cet oral. En effet, nous avons noté des termes dévalorisant ce français parlé par les étudiants, attribués par ceux qui le jugent assez bon en répondant à la question : «Qu’est-ce qui caractérise ce français parlé par les étudiants ?».

\section{Les caractéristiques du français parlé par les étudiants}

Les enseignants qui pensent que le niveau des étudiants à l'oral est assez bon le caractérisent malgré tout d'une manière dévalorisante. Ils dénigrent dans leurs réponses la façon de parler des étudiants :

«Le français parlé par les étudiants est caractérisé par le non-respect du statut de l'interlocuteur et du cadre formel, ce qui entraîne chez eux le tutoiement, le recours à des registres de langue souvent familiers et vulgaires. »

«Ils tutoient tout le monde, conjugaison souvent erronée, vocabulaire très réduit. »

«Retour fréquent vers la langue maternelle, accent non soigné. »

«Beaucoup d'interférences avec les langues locales. »

"Il est caractérisé par l'abréviation (la troncation). »

«Beaucoup d'interférences. »

«La plupart des étudiants n'ont pas acquis la compétence de l'expression orale (problème de fluidité, d'expressivité, de prononciation). »

«L'utilisation abusive des conjonctions de coordination et des interjections. »

«Une mauvaise articulation des mots, et des phrases incomplètes dans la plupart des cas. »

«Les étudiants s'expriment trop avec des termes non scientifiques. » 
Une seule appréciation positive caractérise ce français parlé par les étudiants, à savoir « $L a$ bonne prononciation et la bonne construction ».

Les 23 autres enseignants se lancent aussi dans cette optique de dépréciation à travers diverses formulations :

«Je ne considère pas la langue qu'ils parlent comme étant du français, c'est un massacre total de cette langue. »

«Des termes trop familiers, voire argotiques, un français qui est largement confondu entre le formel et l'informel; les étudiants font trop souvent recours aux autres langues (kabyle, arabe) en parlant en français. »

«Il existe de nombreuses interférences entre la langue maternelle et le français. L'étudiant pense en kabyle et il fait semblant de parler en français. »

«Un langage courant (parlé dans la rue) sans esprit scientifique et pédagogique. »

«Coexistence de plusieurs langues dans la structuration de leur phrase (parfois confusion dans la prononciation des mots, autrement dit, création d'une autre phonétique). »

«Pour la plupart, c'est un parler hybride, hésitant, lexique et vocabulaire approximatifs ; syntaxe et grammaire aléatoire. »

«Phrases maladroites, énoncés tronqués, distorsions temporelles. »

"Des incorrections, des phrases inachevées et dont le sens est incomplet. »

Nous pouvons retenir à travers ce qui a été rapporté par les enquêtés que le niveau des étudiants de l'Université de Béjaia, tant à l'écrit qu'à l'oral, est déprécié par les enseignants. Cette dépréciation ressort d'ailleurs dans leurs réponses à la question : «Si vous deviez qualifier ce français parlé des étudiants, quel(s) terme(s) utiliseriez-vous ? \ette question est précédée par une autre qui a pour but de viser un public plus large, à travers le principe de dialogisme où nos enquêtés rapportent les différents termes que les gens attribuent au français parlé par les étudiants. Ces divers qualifiants sont souvent repris par nos enquêtés, influencés par le « déjà-dit » ${ }^{6}$.

\section{La dénomination du français parlé par les étudiants}

À partir des deux questions : «Connaissez-vous à titre d'exemples des termes du français parlé par les étudiants ? » et «Si vous deviez qualifier ce français parlé des étudiants, quel(s) terme(s) utiliseriez-vous ? », nous avons recensé 77 termes et expressions utilisés par les enseignants pour qualifier le français parlé par les étudiants, répartis en un total de 107 réponses. Ce sont, par ordre décroissant : 
français cassé (13), français familier (5), langage de la rue (3), français kabylisé (3), français arabisé (3), frankabyle (2), français «du n’importe quoi» (2), français non académique (2), français cassé mal placé (2), français vulgaire (2), français-kabyle (2), argot (2), français populaire (2), français indigne (1), français de rue (1), français de la frime (1), français d'imitation (1), francarabe (1), français algérien (1), français incohérent (1), français incorrect (1), francarouia ${ }^{7}(1)$, langue hybride (1), français artificiel (1), verlan (1), français interlangue (1), français algérianisé (1), français trop familier (1), français mal dit (1), français pas assez bon (1), français très mal parlé (1), français mal soigné (1), français non-maîtrisé (1), français entrecoupé (1), français relâché (1), français coloré (1), français «light »(1), français transformé (1), français massacré (1), français médiocre (1), français bledard (1), français chamboulé (1), mauvais français (1), français approximatif (1), français de défoulage (1), français inqualifiable (1), français parlé (1), français mal parlé (1), français mal structuré (1), français populace (1), français maison (1), français informel (1), français local (1), français fluide (1), français simple (1), français moyen (1), français nouveau (1), français langue étrangère (1), bas français (1), français fragmenté (1), sabir (1), français estudiantin (1), alternance codique (1), français amalgamé (1), français patchwork (1), bon français (1), français chic (1), français classe (1), français à la mode (1), français des «V.I.P» (1), français de salon (1), français TF1 (1), code switching (1), algérianisme (1), français de maman (1), français décomposé (1), kabylo-français (1).

L'observation de ces différents qualifiants fait ressortir une certaine classification. En effet, les expressions sont regroupées selon quatre paramètres, à savoir, par ordre décroissant :

- $\quad$ les désignations relatives à la structure de la langue ;

- $\quad$ les désignations relatives à la qualité et/ou registre de la langue ;

- $\quad$ les désignations référentielles ;

- les désignations relatives à l'usage de la langue.

Les désignations relatives à la structure du français parlé sont au nombre de 44 expressions ${ }^{9}$ sur 107 :

français cassé (13), francarouia (1), français mal structuré (1), français entrecoupé (1), français massacré (1), français chamboulé (1), français fragmenté (1), français patchwork (1), sabir (1), code switching (1), français amalgamé (1), alternance codique (1), français décomposé (1), verlan (1), français kabylisé (3), kabylo-français (1), langue hybride (1), 
français cassé mal placé (2), français arabisé (3), frankabyle (2), français-kabyle (2), français interlangue (1), francarabe (1), français incohérent (1), français coloré (1).

Comme nous pouvons le constater, «cassé » est l'adjectif le plus récurrent. Les enseignants font référence à la structure brisée et fragmentée de ce français parlé par les étudiants, renvoyant ainsi à une utilisation déstructurée de la langue, à travers les adjectifs « fragmenté », «massacré » et « chamboulé ». Ils signalent également un parler issu d'un mélange de deux langues, à savoir le français et le kabyle ainsi que le français et l'arabe.

Les désignations relatives à la qualité et/ou au registre du français parlé sont représentées par les appellations suivantes, qui sont au nombre de 36 :

français familier (5), français trop familier (1), français «du n'importe quoi » (2), français vulgaire (2), argot (2), français incorrect (1), français pas assez bon (1), français mal soigné (1), français relâché (1), français « light » (1), français transformé (1), français indigne (1), français médiocre (1), mauvais français (1), français approximatif (1), français populace (1), français informel (1), français artificiel (1), français fluide (1), français simple (1), français moyen (1), français nouveau (1), bas français (1), français populaire (2), bon français (1), français chic (1), français classe (1), français inqualifiable (1).

La majorité des désignations renvoie au registre familier dans la mesure où les étudiants ne réalisent pas un français soutenu. Le recours à un nom de «sous-langue », à savoir «argot » (avec deux occurrences) en témoigne. Néanmoins, «bon», «classe » et «chic » viennent marquer la perception de la bonne qualité de ce français parlé par les étudiants.

Il y a aussi des désignations référentielles. Par référentiel, nous entendons les termes qui renvoient à un objet du monde, à toute réalité supposée extérieure à soi ou à une situation décrite. Nous avons 22 termes au total :

\section{- En référence à l'ancrage locatif :}

français local (1), français algérianisé (1), français algérien (1), algérianisme (1), français blédard (1), français de maman (1), français maison (1).

Certains enseignants ne comparent pas le français des étudiants avec le français de l'hexagone ; ils considèrent plutôt que c'est un français local, spécifique à l'Algérie. Les qualifiants «français de maman » et «français de maison» renvoient plutôt à l'ancrage familial.

\section{- En référence à une situation :}

français de la rue (1), langage de la rue (3), français de salon (1), français des «V.I.P»(1), français à la mode (1), français TF1 (1). 
Nous observons une opposition distincte entre des désignations qui renvoient à l'espace où le français est considéré comme situationnel (formel et informel), à savoir « rue » par opposition à « salon », «TF1», «VIP » et « mode ».

\section{- En référence à un comportement :}

français d'imitation (1), français de défoulement (1), français de la frime (1).

L'un des enquêtés trouve que les étudiants font de l'excès de zèle dans la mesure où ils essayent d'imiter le français parlé à la télévision. Un autre qualifie les étudiants de frimeurs et un troisième signale le caractère émotionnel qui donne à l'étudiant le sentiment de plaisir en parlant le français.

\section{- En référence à une réalité constatée :}

français non académique (2), français langue étrangère (1), français estudiantin (1).

Deux enseignants estiment que les étudiants doivent parler un français académique. Deux autres considèrent que les étudiants ont, avant tout, affaire à une langue étrangère et que le parler qui en résulte, n'est que la conséquence de leur apprentissage.

Les désignations relatives à l'usage sont regroupées dans les expressions suivantes : français non-maîtrisé (1), français mal parlé (1), français très mal parlé (1), français mal dit (1), français parlé (1).

Nous remarquons dans ce regroupement une confusion de la part des enquêtés entre "parlé » qui signifie « usage » et «mal parlé » qui renvoie à la qualité. Pour eux, le français parlé est mal vu.

Le nom que l'on choisit pour désigner une chose, un être ou un concept peut contribuer à en donner une impression positive ou négative. Il y a donc des noms qui sont favorables, positifs et qui ont une connotation méliorative, mais aussi des noms qui sont défavorables, négatifs et qui ont une connotation péjorative. Ce qui ressort de notre analyse c'est que la majeure partie des enseignants, qu'ils soutiennent l'idée que les étudiants sont assez bons ou qu'ils sont mauvais à l'oral, qualifie péjorativement ce français parlé. Nous relevons des représentations peu flatteuses et plusieurs dénominations péjoratives le recouvrant, mettant en cause sa pratique, avec une prédominance spectaculaire d'adjectifs à connotation péjorative. Nos enquêtés usent de qualifiants divers et variés pour désigner le niveau des étudiants en langue française. Ils insistent sur le fait que le français parlé par les étudiants est loin de répondre aux exigences de la norme et du français de référence. Ils distinguent ainsi le parler des étudiants du parler d'autres locuteurs à travers un processus de catégorisation. 
En effet, à quelques exceptions près, nos étudiants parlent mal le français et éprouvent d'énormes difficultés à s'exprimer devant leurs enseignants. C'est la raison pour laquelle ils préfèrent, pour faire passer leur message, employer une langue qui leur est spécifique avec une déformation parfois exagérée de la syntaxe; ils pensent en kabyle et en arabe et ils s'expriment en français. Le problème de la prononciation est encore plus dramatique, ce qui rend parfois leur discours inintelligible. Ce français est considéré par les enseignants comme appartenant, tout simplement, aux étudiants.

Ce phénomène ne concerne pas seulement les étudiants de Béjaia, mais se répand aussi dans toutes les universités algériennes. Néanmoins, nous notons quelques différences dans la maîtrise du français, liées à la stratification sociale et géographique ainsi qu'aux représentations du français chez les locuteurs (étudiants mais aussi enseignants). Les gens du Nord arrivent à parler un français phonétiquement correct, contrairement aux gens du Sud. Comme le souligne Nabila Bedjaoui, «Les Algériens du Sud ont leur propre image de cette langue. A force de ne pas la maîtriser, ils la perçoivent comme étant étrange, difficile voire impossible à pratiquer » (11). Dans son article, elle montre que le français parlé par les gens du Sud est différent et que cette différence s'amplifie de plus en plus. Elle souligne :

Les apprenants ont de réels problèmes au niveau de la prononciation de certains phonèmes, ce qui déforme les sons et fait aboutir à des mots autres que les mots. A force de mal prononcer le français et ceci pendant des années, ils se trouvent dans l'incapacité de le parler correctement. Leur appareil phonatoire ne fonctionne plus que dans ce sens faux. Les organes le constituant semblent être déformés. (21-22)

La question qu'elle se pose, est : «sommes-nous témoins de l'émergence d'une nouvelle forme de français, une variation ? Ou bien s'agit-il d'un changement linguistique en cours, sachant que ce changement ne peut-être que la conséquence d'un changement social (...) résultat de l'arabisation ?» (22)

D'autres études ont montré qu'en raison de facteurs historiques, sociolinguistiques, etc., le français en usage en Algérie diffère de celui de France. Brahim Kethiri s'est aussi intéressé aux locuteurs du Sud mais en s'interrogeant sur d'autres usagers de la langue française «qui utilisent et acceptent un français qui intègre les emprunts aux idiomes locaux en contact»(245). Dans son article, en s'appuyant sur les résultats d'un questionnaire proposé à des professeurs de l'enseignement secondaire de français langue étrangère, il s'est interrogé sur le recours à l'emprunt dans les classes de langue et sur la tolérance de ces professeurs à l'égard de ces termes d'origine arabe et berbère :

Les enquêtés, tout en déclarant que le fait de recourir aux emprunts demeure un procédé équivoque à la limite de l'inutile, reconnaissent recourir pourtant à ce 
procédé dans des situations que la pratique de la classe leur impose (cas de synonymie, explication de mots nouveaux pour les élèves...). Ils diffèrent ainsi de leurs prédécesseurs des décennies 1960 et 1970 qui ne se permettaient guère l'usage de ces termes en classe de français et sanctionnaient parfois sévèrement leurs élèves quand ceux-ci s'ingéniaient à le faire. Ces professeurs d'antan avaient, selon les sujets enquêtés, la prétention d'être pour les élèves les vecteurs vivants de la norme du français, norme fondée sur l'usage des écrivains. (247)

Ce recours aux emprunts pourrait s'expliquer par

la composition du corps professoral, qui comporte à la fois des professeurs issus de l'école fondamentale où la langue d'enseignement est l'arabe, et des enseignants pour qui la langue française a été langue d'enseignement (...) [ajouté à cet élément] le niveau médiocre des élèves en langue française, tant décrié par la plupart des professeurs de la région, [justifiant cette méthode] qui tient compte de l'invasion du français local, par exemple dans les médias, par des termes provenant des langues en présence dans le marché linguistique algérien. (248)

Arrivés à l'université, ces étudiants déjà initiés et habitués à l'usage d'un français spécifique représentant des écarts qui affectent la langue, ne font aucun effort pour améliorer leur niveau et s’approprier les normes du français de référence.

\section{Bibliographie}

Bedjaoui, Nabila. «L'apprenant du français dans le Sud algérien entre une langue étrangère et l'étrangeté d'une langue ». Revue de la faculté des lettres et langues 10-11 (2012): $11-23$.

Benmayouf, Yamina-Chafia. «Le nom de l'arabe dans l'Algérie contemporaine ». Les enjeux de la nomination des langues dans l'Algérie contemporaine. Dir. Foudil Cheriguen. Paris : Harmattan, 2007. 71-90.

Canut, Cécile. «A la frontière des langues. Figures de la démarcation ». Cahiers d'études africaines. Langues dédiées (2001) : 163-164.

Cheriguen, Foudil. «Quels mots pour dire 'démocratie' dans l'Algérie d'aujourd'hui ?» Langage et politique. Dir. André-Marcel d'Ans. Paris : Didier, 1995. 215-235.

---. « Politique linguistique en Algérie ». Mots 52 (1997).

---. Les mots des uns, les mots des autres : le français au contact de l'arabe et du berbère. Alger : Casbah, 2002.

Gadet, F. « Le français tel qu’on le parle ». Le langage Hors-série 27 (1999). 
Grandguillaume, Gilbert. "Arabisation et légitimité politique en Algérie ». Langues et pouvoir, de l'Afrique du Nord à l'Extrême-Orient. Dir. Chaker Salem. Aix-enProvence : Edisud, 1998. 17-23.

Hamers, Josiane F. et Michel Blanc. Bilingualité et bilinguisme. Bruxelles : Mardaga, 1983.

Kethiri, Brahim. «Les professeurs de français en Algérie, vecteurs légitimes de la norme et l'emprunt aux idiomes locaux ». www.unice.fr/ILF-CNRS/ofcaf/19/kethiri.pdf. 1999. 245-259. Consulté 13/03/2013.

Queffélec, A. et al. Le français en Algérie, lexique et dynamique des langues. 1e éd. Bruxelles : De Boeck, Larcier et Duculot, 2002.

Rapport général de la commission de réforme du système éducatif algérien (projet), mars 2001.

\section{Notes}

${ }^{1}$ Il s'agit de l'arabe dialectal, bien sûr.

2 « Le contact de deux langues telles que l'arabe et le français a donné naissance à une sorte de transplantation de la langue qui va au-delà du simple emprunt. C'est une sorte de bilinguisme qui, grâce à un mélange de termes arabes et français dans les limites d'une même phrase a été pratiqué. Ce parler bilingue se maintient surtout chez les autochtones cultivés qui emploient des termes locaux quand le référent est une réalité locale, des termes français quand le référent renvoie à une réalité technique et scientifique ou même institutionnelle » (Cheriguen, Mots des uns, 11)

${ }^{3}$ Un deuxième campus a ouvert ses portes en 2003-2004, où sont transférées du premier pôle la faculté des lettres et des sciences humaines ainsi que la faculté de droit, la faculté des sciences économiques, sciences de gestion et sciences commerciales. La faculté de médecine a ouvert ses portes depuis 2007-2008.

${ }^{4}$ Nous avons regroupé dans notre analyse les deux catégories «bon » et « assez bon » dans « assez bon ».

${ }^{5}$ Nous avons regroupé «mauvais » et « très mauvais » dans «mauvais ».

${ }^{6}$ Comme le souligne Cécile Canut : «Comme dans toute situation langagière, c'est le positionnement de soi par rapport à l'autre qui est en jeu et se manifeste par différentes distanciations dans les discours et une appropriation variante des discours de la doxa. Cette grande variation s'inscrit dans ce que Bakhtine appelle le dialogisme et que l'on entend aujourd'hui par interdiscursivité, c'est-à-dire les discours antérieurs, le "déjàdit» ou le "dit avant», "ailleurs", qu'ils soient politiques, idéologiques, sociaux, etc., parmi lesquels s'opèrent parfois des choix, et qui sont consciemment ou inconsciemment réactualisés dans nos propres discours $»(163-164)$.

${ }^{7}$ L'enseignant souligne dans son explication que «francarouia » est composé de « français » et de « iroui » du kabyle « entremêlé ».

${ }^{8}$ Néologisme : un jeu sur la morphologie nominale (remplacement du suffixe-ment par le suffixe-age).

${ }^{9}$ Nous avons mentionné entre parenthèses la fréquence des désignations. 